\title{
The immediate effect of transcranial direct current stimulation combined with peripheral electrical stimulation in the control of temporomandibular pain in subjects with sickle cell disease: A protocol for one session randomized, crossover, double-blind clinical trial
}

\section{Lilian Anabel Becerra de Oliveira' (1) Tiago da Silva Lopes ${ }^{2}$ (1) Abrahão Fontes Baptista ${ }^{3}$ (1) Katia Nunes Sá 4 (1)}

${ }^{1}$ Corresponding author, Faculdade Adventista da Bahia (Cachoeira), Escola Bahiana de Medicina e Saúde Pública (Salvador). Bahia, Brazil. lilian.becerra@adventista.edu.br ${ }^{2}$ Faculdade Adventista da Bahia (Cachoeira), Universidade Federal da Bahia (Salvador). Bahia, Brazil. tslopes.physio@gmail.com ${ }^{3}$ Universidade Federal do ABC (São Bernardo do Campo). São Paulo, Brazil. abrahao.baptista@gmail.com ${ }^{4}$ Escola Bahiana de Medicina e Saúde Pública (Salvador). Bahia, Brazil. katia.sa@gmail.com

ABSTRACT | INTRODUCTION: Temporomandibular disorder (TMD) is currently considered a central sensitization syndrome that belongs to the orofacial nociplastic pain group and offers great clinical practice challenges. It can also be identified in individuals with sickle cell disease. Neuromodulation is a promising therapy that can help individuals with chronic refractory pain. To our knowledge, there is no treatment proposal for these individuals with chronic orofacial pain resulting from sickle cell disease. OBJECTIVE: This is a protocol of a randomized, double-blind, cross-over clinical trial. The purpose of this protocol is to investigate whether the immediate effect of transcranial direct current stimulation can be increased by adding the effect of peripheral sensory electrical stimulation. METHODS: Twenty women between 18 and 49 years of age will be screened to participate in this cross-over study, where they will all receive the three types of a protocol with a oneweek washout. Active transcranial Direct Current Stimulation (tDCS) + active Peripheral Electrical Stimulation (PES); Active tDCS + PES sham and tDCS sham + PES sham. Stimulation with tDCS will be at $2 \mathrm{~mA}$ anodic over the motor cortex for 20 minutes ipsilateral to the most painful temporomandibular joint (TMJ). Peripheral electrical stimulation will be at $100 \mathrm{~Hz}$ over the most painful TMJ masseter muscle for 30 min. OUTCOME: The main outcome will be pain intensity assessed by a VAS scale and a pressure algometer in grams. Endogenous pain modulation will be analyzed through the temporal summation of pain with Aesthesio precision tactile sensory filaments and conditioned pain modulation (CPM) evaluated by an algometer and thermal conditioned stimulus as secondary outcomes. Data will be analyzed using ANOVA of repeated measures, controlling for confounding variables.

KEYWORDS: Neuromodulation. Endogenous pain modulation. Sickle cell disease. Temporomandibular dysfunction. 
Due to several events of vaso-occlusive crisis, individuals with Sickle Cell Disease (SDC) are more likely to develop musculoskeletal pain; thus, approximately $30 \%$ of adults suffer chronic pain ${ }^{1}$. Besides, studies have shown that chronic pain has a potential impact on daily life. Psychological discomfort, physical dysfunction, and functional limitation may further limit individuals' quality of life with SCD. Therefore, there is an increasing awareness that chronic pain is part of the experience of individuals with $\mathrm{SCD}^{2}$.

Among the painful musculoskeletal manifestations in individuals with SCD, Temporomandibular Disorder (TMD) is classified as Central Sensitivity Syndrome (CSS) and therefore is incredibly challenging. The CSS is a category of interrelated disorders with Central Sensitization (CS) as a common etiology ${ }^{3}$. $\mathrm{CS}$ is a physiological phenomenon involved in the nociceptive process 4 . However, this phenomenon's persistence has been associated with maladaptive plasticity in the central nervous system, which can include dysfunction in modulatory control of pain in descending and/or ascending pathways ${ }^{5}$. In addition, a study showed that there is an association between dysfunction in modulatory control of pain in descending and intracortical inhibition ${ }^{6}$.

Maladaptive plasticity resulting from central sensitization has been identified in SCD and seems to play an essential role in maintaining and chronicity in this population ${ }^{7}$. A recent study showed that high central sensitization had been associated with pain and clinical measures, such as more insufficient sleep, higher situational catastrophizing, higher frequency of vaso-occlusive crisis, and pressure pain threshold in SCD individuals ${ }^{\circ}$. In addition, SCD individuals with chronic pain compared to those without chronic pain showed significant differences in functional connectivity between the periaqueductal gray (PAG) and in pronociceptive brain regions $s^{7,9}$.
Neuromodulation through electric currents is one way to interfere with cortical plasticity related to chronic pain. Among the available interventions to modulate neuronal activity with electric currents is Transcranial Direct Current Stimulation (tDCS). The tDCS is effective as a single-phase current that induces changes in polarity-dependent excitability in the motor cortex, plasticity induction, functional modulation within the primary motor cortex M1 10,11 .

The mechanism of action of tDCS includes neuromodulatory (during application) and neuroplastic (after application) effects. At first, the effect is related to changes that occur in the neuronal membrane's resting potential. Anodic stimulation initiates cell membrane oscillations that exhibit high frequency and low amplitude characteristics during depolarization. The effect's duration depends on protein synthesis, accompanied by intracellular changes and calcium levels ${ }^{12}$. The effects of a single application of tDCS on corticospinal excitability depend on the intensity of the stimulus and its duration. A study by Nietsche MA, Paulus W, the same authors showed that 13 minutes of anodic stimulation with tDCS on M1 caused corticospinal excitability up to 90 min after the session ${ }^{13}$. Therefore, a tDCS session may be useful for generating data that may suggest more prolonged use of this technique.

Studies show that tDCS alone has a moderate (20$40 \%$ ) effect on pain control14; however, it has been proposed as an association of techniques to increase this effect $\frac{15}{5}$. These therapeutic associations assume that brain responsiveness to a particular therapy can be facilitated by another that alters cortical excitability. Peripheral Electrical Stimulation (PES) is a neuromodulatory technique that can produce cortical excitability changes, depending on stimulation intensity, frequency, and duration. Inversely the PES sensory threshold decreases excitability, whereas the motor threshold has the opposite effect ${ }^{16}$. 
When two excitatory stimuli are associated, a null response can occur due to meta-plasticity. However, the association between inhibitory and excitatory stimuli can also result in a synergistic effect ${ }^{17}$. Therefore, PES at a sensory threshold associated with anodic tDCS produces a summing analgesic effect. For example, Boggio ${ }^{15}$ experienced a decrease in pain intensity by $36.5 \%$ with the two techniques' union, when tDCS alone resulted in only a $15 \%$ decrease in pain.

Finally, maladaptive brain plasticity may suggest chronic pain and explain why some individuals with SCD display refractory pain and are not responsive to pharmacological and non-pharmacological analgesic treatment ${ }^{18}$. It is why new treatments are necessary. There are already new proposals for using neuromodulation for chronic pain of SCD patients, but results have not been published ${ }^{19}$ and are not focused on TMD. Therefore, these studies provide a scientific basis for the reasoning behind cortical stimulation in the presence of orofacial pain in patients with SCD.

\section{Objectives}

\section{Primary Objective}

1. To determine the immediate effect of anodic tDCS and sensory PES on chronic myofascial and atherogenic TMD pain intensity in sickle cell disease individuals.

\section{Secondary Objective}

1. To determine the neuromodulatory effect of anodic tDCS and PES stimulation on endogenous pain modulation in individuals with chronic myofascial and atherogenic TMD.

\section{Materials and methods}

\section{Methods: Study design, participants, interventions, and outcomes}

It is a clinical, crossover, controlled, block-randomized, double-blind trial. To determine the immediate effect of anodic tDCS on M1 and sensory PES on myofascial and atherogenic TMD pain intensity in individuals with sickle cell disease with different combinations and determine the neuromodulatory effect of anodic tDCS and PES stimulation on endogenous pain modulation.

The participants of this study will be recruited among patients with SCD registered in the Basic Unit of Health of their neighborhood of the 31 st Regional Health Directorate (DIRS-BA). They will be evaluated for TMD and be interviewed on their willingness to participate in the study. Next, three encounters will be scheduled in the Bahia Adventist College's Academic Clinic in Cachoeira - Bahia, Brazil.

\section{Inclusion criteria}

A. Women between 18-49 years old with SCD diagnosis and TMD with 3 months or more of myofascial pain over the temporomandibular joint.

B. Pain intensity above 3 to 10 points of visual analogic scale (VAS).

C. Women with SCD of genotype HbSS or HbSC only.

\section{Exclusion criteria}

A. Any contraindication to using tDCS or PES such as cochlear implants, cardiac pacemaker or metal implants in the skull/brain, pharmaceuticals that modify the threshold of neuronal activation (i.e., antidepressant, anticonvulsant, and antipsychotic); the history of seizure or epilepsy; and pregnancy. 
B. Metal implants in the PES site.

C. Occurrence of infectious disease in the week before inclusion in the study.

\section{Criteria for discontinuity}

A. A moderate adverse effect, uncomfortable enough to interfere with the patient's usual activities.

The potential adverse effects described by the literature are Itching, tingling, skin redness, somnolence, concentration issues, headache, fatigue, lightheadedness, and skin burning under the electrode (rare). The intensity of any of these adverse effects depends on the subject's sensitivity, defining the decision to continue or not in the study ${ }^{14}$.

B. Participant withdraws consent at any stage of the study.

\section{Individuals who will perform interventions}

Researcher 1: will do the screening and enrollment, will apply the sociodemographic and specific questionnaires.

- Catastrophic thoughts about pain (BP-PCS)

- Brazilian Portuguese Central Sensitization Inventory (BP-CSI)

- Hospital Anxiety and Depression Scale (HADS)

- Brief Pain Inventory (BPI) to Brazilian patients

- Short Form Health Survey 36 (SF-36)

- Duke's religiosity index

This researcher will give the questionnaires to the participants in a silent room of the Basic Unit of Health of their neighborhood. 45 minutes will be allotted for this questionnaire. Then will schedule the next session in the clinical setting.

This researcher will have the function of evaluating before and after the intervention, ignoring what protocol the patient underwent. This function will include applying the Research Diagnostic Criteria for the Temporomandibular Disorders RDC/TMD, the evaluation of Temporal Summation of Pain (TSP), and Conditioned Pain Modulation (CPM). This process will have 30 minutes lasting and reviewed in one room of the Academic Clinic.

Assistant Researcher 1: On day two, this researcher will have the duty to receive all participants at the Academic Clinic's front desk and will lead them to the room of researcher 1 for pre-evaluations. Then will guide the participants to assistant researcher 2 for randomization and intervention. Finally, it will guide the participants back to researcher 1 for the post-tests. Assistant researcher 1 will not have access to the database or randomization until the end of the research.

Assistant Researcher 2: This researcher will have the function of conduct computational randomization and will apply intervention as randomization indicates. Active tDCS + active PES or active tDCS + sham PES or sham tDCS + sham PES. After completing the treatment, a questionnaire on safety will evaluate the immediate adverse effects. The second and third day of treatment was scheduled by Assistant Researcher 2 in advance by telephone, and they asked if there was a moderate adverse effect that affected the individual's daily activities. If positive, the treatment will be discontinued. Any other mail effect will be registered. Only assistant researcher 2 is to know the treatment offered to the participant in the crossover process. This process will be completed in the Academic Clinic and will take about 40 minutes.

Researcher 2: This researcher will have the function to process the data in spreadsheet excel and do inferential analysis in SPSS

\section{Intervention}

This study will be developed in four steps (Figure 1), divided into four days with a one-week washout. On the first day, the screening procedures and sample characterization will be performed. In the next three days, randomization and intervention protocol will be performed, having before and after three evaluations (RDC/TMD, TSP, and CPM), obeying randomization allocation. (Figure 2). 


\section{$\mathrm{X}$ 두}

Evaluations for elegibility In Basic Health Unit

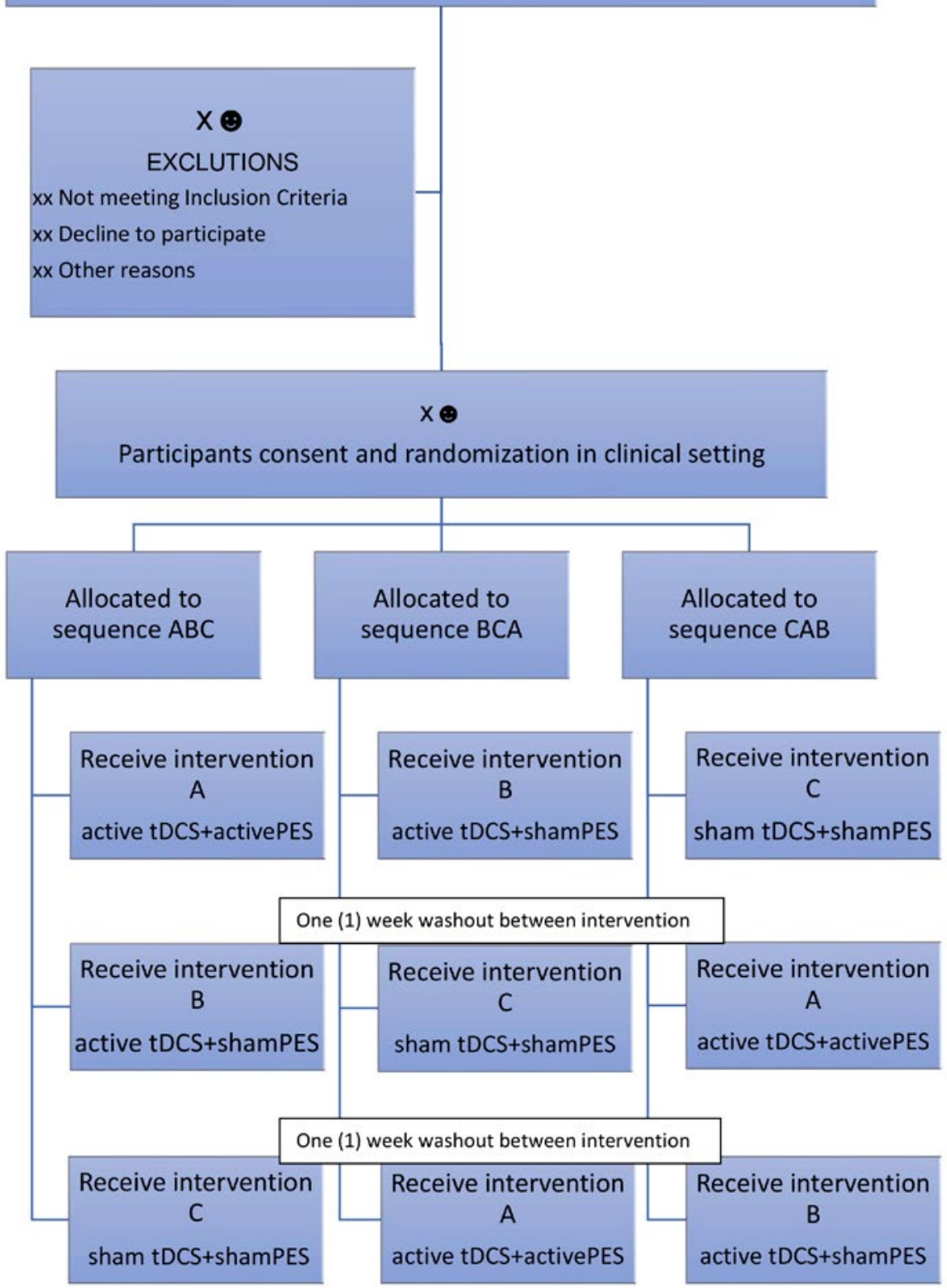




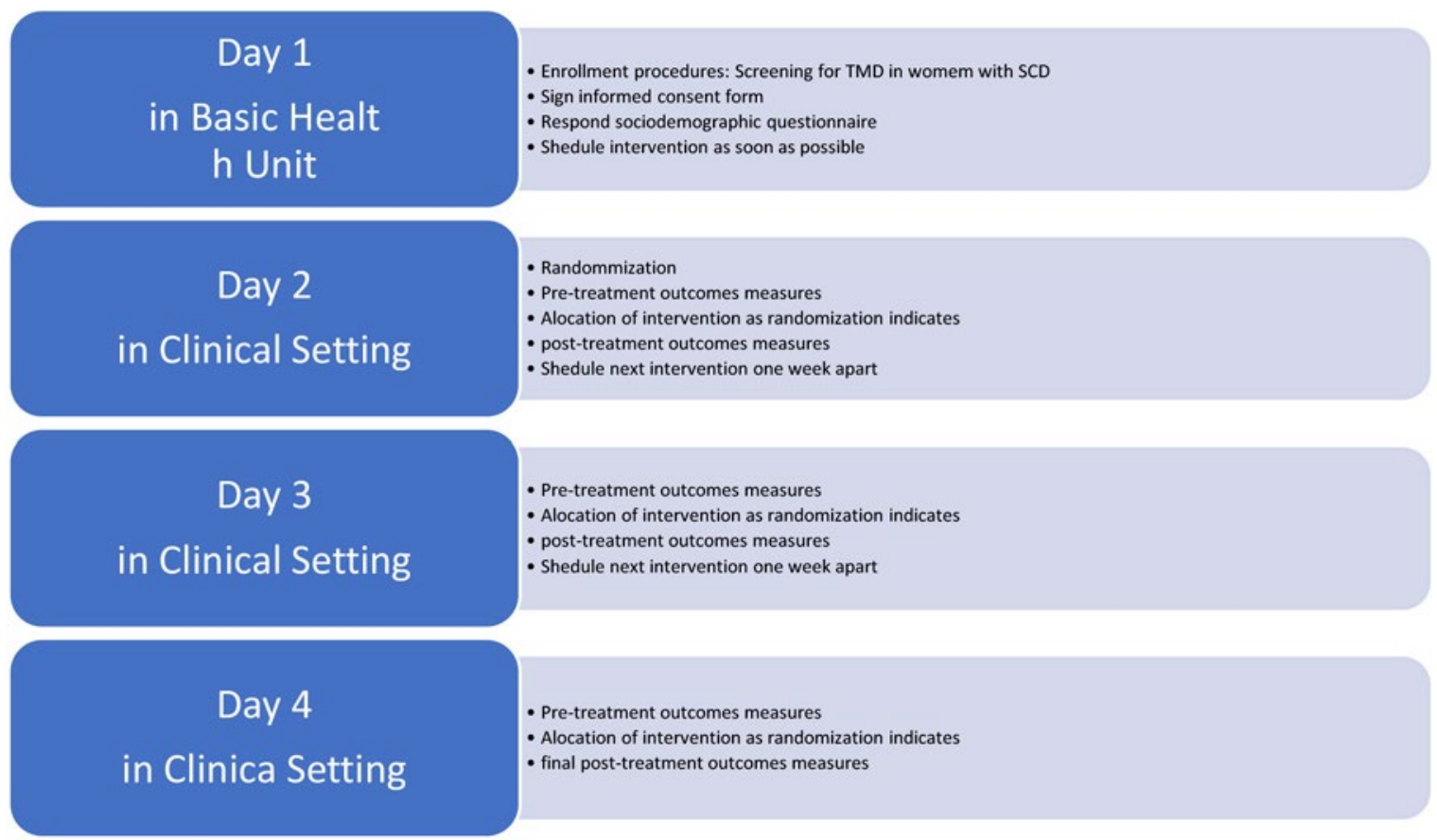

\section{Day 1}

The participant with SCD diagnosis will be invited to undergo screening at their local Basic Health Unit. Researcher 1 will guide the participant in a quiet room to explain the goals of the research. In the first step, we will identify women with SCD who also have pain secondary to temporomandibular dysfunction. An informed consent form will then be read and signed. Next, an evaluation will be conducted, and the questionnaires answered. Common psychological pathologies may interact with complications associated with SCD; this is why it has been recommended to screen these comorbidities using standardized screening tools $\mathrm{s}^{20,21}$.

A. The sociodemographic questionnaire gathers information on age, education level, profession, marital status, race, religion, and pain levels in the temporomandibular joint. The type of sickle cell disease is also recorded; information is taken from a medical statement (printed or written manually) that the subject presents for this first interview. Inclusion and exclusion criteria are assessed at the beginning of the interview.

B. Brazilian Portuguese Pain Catastrophizing Scale (BP-PCS) - This questionnaire consists of 13 items evaluating self-reported catastrophizing thoughts, feelings, and behaviors when one is in pain ${ }^{22}$.

C. Brazilian Portuguese Central Sensitization Inventory (BP-CSI). Is a self-report questionnaire of health symptoms. It is designed as an easy-to-administer screen for patients at high risk of having central sensitization and helps classify chronic pain patients. It consists of 25 questions with five possible answers from never to always. It is an ordinal scale, and each response has one value from zero to four ${ }^{23}$.

D. Hospital Anxiety and Depression Scale (HADS) comprises 14 self-reported questions that are divided into two subscales. The subject will rate each item using an ordinal scale varying from zero (non-existent symptom) to three (very severe symptom) ${ }^{24}$. 
E. Brief Pain Inventory to Brazilian patients (BPI) consists of nine items that are arranged in two dimensions: the intensity of pain and the impact of pain in the patient's life. The BPI asks patients to rate their pain intensity and the pain interference with general activities, mood, walking ability, regular work, relationships with others, sleep and enjoyment of life in an 11-point scale ranging from zero (no pain) to ten (as bad as it can be). It includes a corporal diagram to assess pain location, measures the percentage of pain relief, and asks patients to describe which treatments are being used to control pain. A high score represents a high pain intensity or pain interference ${ }^{25}$.

F. Short Form Health Survey 36 (SF-36) comprises 36 multidimensional questions that will give a raw scale of eight different concepts as functional capacity, physical aspects, pain, general health, vitality, social aspects, emotional aspects, and mental health. It has a final score from zero to 100 , where zero corresponds to the worst general health and 100 the best general health $\underline{26}$.

G. Duke's religiosity index is a five-item scale measuring three dimensions of religious involvement related to health outcomes. Organizational religiosity, non-organizational religiosity, and intrinsic religiosity. It is recommended that the values are not summed but analyzed separately ${ }^{27}$.

\section{Day 2 First moment}

The participant will check-in at the Academic Clinic front desk and receive the assistant researcher 1 . Assistant researcher 1 will document the following: caffeine intake, medications taken in the last 24 hours, menstrual state, and hour of the day. Caffeine intake will be classified into three categories: low (<100 mg/day), moderate (101-200 mg/day), or high (>201 mg/day). The menstrual state will be divided into: menstrual, follicular, periovulatory, luteal, and premenstrual. Next, the patient will be guided to researcher 1, an experienced physical therapist, to review three evaluations.

A. ResearchDiagnostic CriteriaforTemporomandibular Dysfunction (RDC/TMD). This instrument consists of a detailed evaluation of the stomatognathic system involved in the conditions of temporomandibular disorder. It consists of two axes. Axis I evaluate the clinical aspects with ten questions. Axis II psychosocial aspects, with 31 questions. In this diagnostic regimen, the patient is framed in 3 groups: Group I - Muscular Disorders; Group II - Disk Displacement; Group III Other Joint Conditions ${ }^{28,29}$. Currently, this instrument has a new version called Diagnostic Criteria for Temporomandibular Dysfunction (DC/TMD) ${ }^{30}$. However, it has not been validated for the Portuguese Brazilian population.

B. Endogenous Pain Modulation Testing. Patients will be seated in a comfortable armchair in a quiet room with an ambient temperature of $23^{\circ} \mathrm{C}$.

a. To evaluate pain facilitation, the Temporal Summation of Pain (TSP) will be tested with Aesthesio precision tactile sensory evaluator filaments.

i. Evaluation of the mechanical detection threshold of each individual. A pinprick instrument was chosen from a custom-made weighted set of 20 calibrated instruments (Aesthesio, USA, DanMIc global, LLC, revised May 2017, 0.2 mm diameter flat contact surface, target force from $0,008 \mathrm{~g}$ to $300 \mathrm{~g}$ ). The selected instrument will be held in a vertical position perpendicular to the skin surface to apply three stimuli, over the skin of the thenar region of the dominant hand and the masseter region of the most painful side. Participants will be instructed to verbalize when they feel the stimulus. The stimulus will end once the filament bends-approximately two seconds. It will be considered a mechanical detection threshold that is perceived at least two out of three times. Each patient will be asked to keep their eyes closed.

ii. Evaluation of pain detection. The test uses over four levels of the pinprick instrument registered as a mechanical detection threshold. Each patient will be asked to declare a sensation of pain, one to ten, on the Visual Analogic Scale (VAS). Zero, being no pain, and ten, the most insupportable pain felt. After identifying and registering this threshold, testing will continue to detect pain type three of VAS. 
iii. Temporal Summation. TSP will be performed according to a standardized protocol ${ }^{31}$ using pinprick stimuli that provoke pain level three on the VAS scale over the skin of the thenar eminence of the dominant hand and the facial skin overlying the middle of the masseter muscles, on the painful side (TMJ). Following a ten-second interval, a series of ten pinprick stimuli will be delivered over a 1-2 cm area near the single stimulus site at $1 \mathrm{~Hz}$ frequency determined using a metronome, and the perceived pain elicited by 10-stimuli series will be rated by the participant and registered by the researcher.

b. Pain inhibition will be assessed by the conditioned pain modulation (CPM) paradigm ${ }^{32}$. First, a test stimulus by an algometer will induce pain of intensity 3 on the VAS (Dukan CTS Gauge, George Medial Hood River, OR. Pat. $N^{\circ} 5301683$, USA) over the region of the long extensor carpialis muscle, next to the lateral epicondyle of the dominant side. Then the participant will be asked to place their non-dominant hand in a stainless-steel container with $1 \mathrm{Lt}$. of hot water at $46^{\circ} \mathrm{C}$ for one minute; this will be the conditioned stimulus. In order to follow the curve of the inhibition mechanism, the test stimulus is reevaluated immediately after the conditioned stimulus and at 30,60, and 90 seconds.

\section{Day 2 Second moment}

Assistant Researcher 1 will guide the participant to Assistant Researcher 2, who will then conduct computational randomization for all sessions. The intervention will be held as randomization indicated. Active tDCS + active PES or active tDCS + sham PES or sham $\mathrm{tDCS}+$ sham PES. The room will be at $23^{\circ} \mathrm{C}$, and the patient will have a comfortable chair with arms to sit on.

A. tDCS: will be applied with a constant current stimulator (Microestim Genius transcranial stimulation device - NKL electronic products, Brazil), connected to two $5 \times 7\left(35 \mathrm{~cm}^{2}\right)$ silicone sponges. Salinemoistened electrodes $(0.9 \%)$ will be positioned on the scalp according to the 10-20 electroencephalography system ${ }^{30}$, with the anode in the individual's primary painful C3 or C4 motor area and the cathode in the supra region. supraorbital contralateral orbital. The $2 \mathrm{~mA}$ stimulation will last for $20 \mathrm{~min}$ with the rise and fall of the 30s. The researcher will ask if there is any bothersome sensation during the trial and will wet the silicone sponges every 5 minutes.
B. PES: A pulsed generator (Neurodyn II, Ibramed, Sao Paulo, Brazil) will be used to administer the PES. Will be used $2.0 \mathrm{~cm}^{2}$ disposable electrodes located in the TMJ region and masseter muscle. Stimulus intensity will be maintained at a sensory threshold of $100 \mathrm{~Hz}$ and a pulse duration of $200 \mu$, with a total duration of 30 minutes.

C. The safety questionnaire will be administered by Assistant Researcher 2.

After finishing this process, Assistant Researcher 1 will guide the patient to Researcher 1 to repeat the evaluation process explained in Day 2 first moment, finishing Day 2.

\section{Strategies to improve adherence to intervention protocols, and any procedures for monitoring adherence}

To improve adherence to the protocol, at first contact and during all the processes, all participants will receive a card (brochure) to register the intensity of pain and acute pain in the morning and evening. This brochure will have our contact number in case they wish to ask any questions. All participants will receive financial aid for round trip transportation, as well as a meal voucher. They will also receive SCD care recommendations and genetic counseling. In the end, all participants will be offered 5 sessions of the most effective intervention for their pain.

\section{Relevant concomitant care and interventions that are permitted or prohibited during the trial}

Individuals who participate in the trial need to have had 15 days without acute pain that resulted in a hospital visit. In addition, they need to be without pain medication for at least 24 hours. All other medications used will be registered.

\section{Potential for adverse effects and damage}

tDCS can generate relatively subtle, self-limiting, and short-term adverse effects that include a mild tingling sensation, itching, burning, mild pain under the electrodes' surface, fatigue, and sleepiness. All of these potential adverse effects can be avoided through appropriate training in the management of the technique. Besides, these adverse effects will be monitored with a specific questionnaire. 
Every participant will be adequately informed about these risks when recruitment and declaration of free and informed consent. The academic clinic comprises physiotherapists and physicians, and they all will be accessible to assist in case of any risk of harm to the participant in this study.

\section{Outcomes}

\section{Primary Outcome Measure}

Pain intensity will be measured at the temporomandibular joint by algometer in kilograms and by the VAS being zero no pain and 10 the most pain imaginable, before and after each treatment.

\section{Secondary Outcomes Measures}

Endogenous pain modulation measured with Temporal Summation of Pain and Conditioned Pain Modulation. Identified with Aesthesia precision tactile sensory evaluator in grams for TSP and registered in grams with algometer for CPM. The amplitude of mouth opening at the pain limit and maximum mouth opening. The number of tender points on the body, the intensity of pain in muscles of mastication.

\section{Participant timeline}

Enrollment of participants will take place between October 2019 and July 2020. All participants will be identified at the Basic Health Unit of cities of the Bahian Recôncavo. It is the geographical region located around Todos-os-Santos Bay, covering the coast and the entire inland region surrounding the Bay. It has been identified as the region with a higher population of SCD individuals in Brazil ${ }^{33}$. (Figure 1)

\section{Sample size}

The sample size was calculated using the G-Power software version 3.1.9.2. The study's primary objective is to test the hypothesis that the therapeutic combination of anodic tDCS in the primary motor cortex and peripheral electrical stimulation at the sensory level promotes a more significant analgesic effect on pain intensity in individuals with DF and TMD after a single session. The sample estimate was based on a randomized sample equally in three crossover groups, two measures (pre- and post-intervention), $80 \%$ power, $5 \%$ alpha, and 0.35 effect size on the primary pain intensity outcome. Using these parameters, the sample was estimated at 20 individuals.

\section{Assignment of interventions and blinding}

Twenty women will participate in this trial. Since this is a randomized crossover trial, the women will receive the three possible treatments to compare results between them. The order of the allocation will be randomized with a computer program, results of the randomization results will be registered and put in a sealed envelope by assistant researcher 1 , who will take care of the information and conduct the treatment protocol.

The sham protocol of the tDCS has been specially programmed for this research by the company (NKL electronic products, Brazil). It has been programmed with $30 \mathrm{sec}$ of the rising ramp, $30 \mathrm{sec}$ of stimulus, $30 \mathrm{sec}$ descent ramp. 20 minutes of sham stimulation, and again, a $30 \mathrm{sec}$ of the rising ramp, $30 \mathrm{sec}$ of stimulus, 30 sec descent ramp.

All participants are blinded for the type of treatment that they will undergo. Researcher 1 and assistant researcher 2 will be blinded as well until the end of the study. Only assistant researcher 1 that will conduct treatment will know each participant's order of allocation and will conduct blind evaluations after each treatment. This evaluation consults adverse effects and asks if the device was turned off or not, and the degree of certainty.

\section{Methods: Data collection, management, and analysis}

Each researcher will do one or more research, but two researchers will not do the same data collection to avoid differences in the collection, as aforementioned.

Researcher 2 will be the only participant of team research that will transcribe all data from the questionnaires on an excel spreadsheet and analyze it with the SPSS program. All questionnaires will be kept safe for ten years. 
The Kolmogorov-Smirnov test will be applied to the continuous variables for analysis of normality. Continuous variables: TMJ pain intensity, age, amount of body tender points, amount of painful masticatory muscles, mechanical detection threshold and pain threshold, temporal pain summation, CPM, mouth opening in millimeter. They will be presented with measures of central tendency and dispersion.

Categorical variables: education, type of sickle cell disease, use of medicines, religion, professional activity, the tendency to depression and anxiety, quality of life, degree of catastrophism, and degree of central awareness. Will be presented in relative and absolute frequencies. The quantitative data used during the sample characterization will be analyzed through one-way ANOVA if the variances are homogeneous. Otherwise, the Kruskal-Wallis test will be used.

To test the neuromodulatory effect of the different therapeutic combinations of anodic tDCS and sensory PES on pain intensity outcomes and endogenous pain modulation, ANOVA repeated measures would be used for each outcome separately if there is normal distribution using the TIME factor. If there is no normal distribution, the Freedman test will be used. All analyses will be performed using the Statistical Package for Social Sciences (SPSS) version 22.0, with a significance level of $5 \%$.

\section{Ethics aspects}

Volunteers will receive an explanation regarding their participation and the freedom to remove consent at any time during the study. They will read and receive answers to any questions before signing an informed consent form (ICF), prepared according to Resolution 466/2012 of the Brazilian National Council of Health. This study has been approved by the Ethics and Research Committee (ERC) of the Faculdade Adventista da Bahia (CAAE No. 94835218.8.0000.0042). The study is registered at REBEC, the Brazilian registration for clinical trial number UTN: U1111-1243-3020. Any changes in the study protocol will be informed to both the ERC and the REBEC. None of the authors acknowledge any potential sources of conflict of interest.

\section{Disclosure}

These trials' results will be shared with the Recôncavo Bahiano local city Basic Health Unit healthcare professionals. The participants are from cities in Recôncavo Bahiano. In addition, each participant will have the opportunity to receive five sessions of the treatment combination that is most effective for their cases. We will also make the results public in an openaccess review.

\section{Acknowledgment}

Lopes TS is financed by Fundação de Amparo à Pesquisa do Estado da Bahia (FAPESB).

\section{Author contributions}

Oliveira LAB wrote the manuscript. Lopes TS, Baptista AF and Sá KN contributed with critical intellectual content.

\section{Competing interests}

No financial, legal, or political competing interests with third parties (government, commercial, private foundation, etc.) were disclosed for any aspect of the submitted work (including but not limited to grants, data monitoring board, study design, manuscript preparation, statistical analysis, etc.).

\section{References}

1. Smith WR, Penberthy LT, Bovbjerg VE, McClish DK, Roberts JD, Dahman B, et al. Daily assessment of pain in adults with sickle cell disease. Ann Intern Med. 2008;148(2):94-101. https://doi. org/10.7326/0003-4819-148-2-200801150-00004

2. Dampier C, Palermo TM, Darbari DS, Hassell K, Smith W, Zempsky W. AAPT Diagnostic Criteria for Chronic Sickle Cell Disease Pain. J Pain. 2017;18(5):490-8. https://doi.org/10.1016/j. jpain.2016.12.016

3. Kindler LL, Bennett RM, Jones KD. Central sensitivity syndromes: mounting pathophysiologic evidence to link fibromyalgia with other common chronic pain disorders. Pain Manag Nurs. 2011;12(1):15-24. https://doi.org/10.1016/j.pmn.2009.10.003

4. Millan MJ. Descending control of pain. Prog Neurobiol. 2002;66(6):355-474. https://doi.org/10.1016/s03010082(02)00009-6 
5. Schaible H-G, Richter F, Ebersberger A, Boettger MK, Vanegas H, Natura G, et al. Joint pain. Exp Brain Res. 2009;196(1):153-62. https://doi.org/10.1007/s00221-009-1782-9

6. Tarragó MGL, Deitos A, Brietzke AP, Vercelino R, Torres ILS, Fregni $F$, et al. Descending Control of Nociceptive Processing in Knee Osteoarthritis Is Associated With Intracortical Disinhibition: An Exploratory Study. Medicine (Baltimore). 2016;95(17):e3353. https://doi.org/10.1097/md.0000000000003353

7. Karafin MS, Chen G, Wandersee NJ, Brandow AM, Hurley RW, Simpson $\mathrm{P}$, et al. Chronic pain in adults with sickle cell disease is associated with alterations in functional connectivity of the brain. PLoS One. 2019;14(5):e0216994. https://doi.org/10.1371/journal. pone.0216994

8. Campbell CM, Moscou-Jackson G, Carroll CP, Kiley K, Haywood Jr C, Lanzkron S, et al. An Evaluation of Central Sensitization in Patients With Sickle Cell Disease. J Pain. 2016;17(5):617-27. https://doi.org/10.1016/j.jpain.2016.01.475

9. Uhelski ML, Simone DA. Sensitization of nociceptors and dorsal horn neurons contributes to pain in sickle cell disease. Neurosci Lett. 2019;705:20-6. https://doi.org/10.1016/j.neulet.2019.04.013

10. Polanía R, Paulus W, Nitsche MA. Reorganizing the intrinsic functional architecture of the human primary motor cortex during rest with non-invasive cortical stimulation. PLoS One. 2012;7(1):e30971. https://doi.org/10.1371/journal.pone.0030971

11. O'Connell NE, Wand BM, Marston L, Spencer S, DeSouza LH. Non-invasive brain stimulation techniques for chronic pain. Cochrane Database Syst. Rev. 2014. http://dx.doi. org/10.1002/14651858.cd008208.pub3

12. Nitsche MA, Paulus W. Excitability changes induced in the human motor cortex by weak transcranial direct current stimulation. J Physiol. 2000;527 Pt 3(Pt 3):633-9. https://dx.doi. org/10.1111\%2Fj.1469-7793.2000.t01-1-00633.x

13. Nitsche MA, Paulus W. Sustained excitability elevations induced by transcranial DC motor cortex stimulation in humans. Neurology. 2001;57(10):1899-901. https://doi.org/10.1212/ wnl.57.10.1899

14. Baptista AF, Fernandes AMBL, Sá KN, Okano AH, Brunoni AR, Lara-Solares A, et al. Latin American and Caribbean consensus on noninvasive central nervous system neuromodulation for chronic pain management (LAC-NIN-CP). Pain Rep. 2019;4(1):e692. https:// doi.org/10.1097/pr9.0000000000000692

15. Boggio PS, Amancio EJ, Correa CF, Cecilio S, Valasek C, Bajwa $Z$, et al. Transcranial DC stimulation coupled with TENS for the treatment of chronic pain: a preliminary study. Clin J Pain. 2009;25(8):691-5. Cited: PMID: 19920718
16. Chipchase LS, Schabrun SM, Hodges PW. Corticospinal excitability is dependent on the parameters of peripheral electric stimulation: a preliminary study. Arch Phys Med Rehabil. 2011;92(9):1423-30. https://doi.org/10.1016/j.apmr.2011.01.011

17. Schabrun SM, Chipchase LS, Zipf N, Thickbroom GW, Hodges PW. Interaction between simultaneously applied neuromodulatory interventions in humans. Brain Stimul. 2013;6(4):624-30. https://doi.org/10.1016/j.brs.2012.09.009

18. New T, Venable C, Fraser L, Rosenberg E, Schmidt J, JamesHerry A, et al. Management of refractory pain in hospitalized adolescents with sickle cell disease: changing from intravenous opioids to continuous infusion epidural analgesia. J Pediatr Hematol Oncol. 2014;36(6):e398-402. https://doi.org/10.1097/ mph.0000000000000026

19. Lopes TS, Lopes TS, Silva WS, Ribeiro SB, Figueiredo CA, Campbell FQ, et al. Does Transcranial Direct Current Stimulation Combined with Peripheral Electrical Stimulation Have an Additive Effect in the Control of Hip Joint Osteonecrosis Pain Associated with Sickle Cell Disease? A Protocol for a One-Session Double Blind, Block-Randomized Clinical Trial. Front. Hum. Neurosci. 2017;11:633. http://dx.doi.org/10.3389/fnhum.2017.00633

20. Pecker LH, Darbari DS. Psychosocial and affective comorbidities in sickle cell disease. Neurosci Lett. 2019;705:1-6. https://doi.org/10.1016/j.neulet.2019.04.011

21. Sójka A, Stelcer B, Roy M, Mojs E, Pryliński M. Is there a relationship between psychological factors and TMD? Brain Behav. 2019;9(9):e01360. https://doi.org/10.1002/brb3.1360

22. Sehn F, Chachamovich E, Vidor LP, Dall-Agnol L, Souza ICC, Torres ILS, et al. Cross-cultural adaptation and validation of the Brazilian Portuguese version of the pain catastrophizing scale. Pain Med. 2012;13(11):1425-35. https://doi.org/10.1111/j.15264637.2012.01492.x

23. Caumo W, Antunes L, Elkfury JL, Herbstrith E, Sipmann RB, Souza A, et al. The Central Sensitization Inventory validated and adapted for a Brazilian population: psychometric properties and its relationship with brain-derived neurotrophic factor. J. Pain Res. 2017;10:2109-22. http://dx.doi.org/10.2147/jpr.s131479

24. Pais-Ribeiro J, Silva I, Ferreira T, Martins A, Meneses R, Baltar M. Validation study of a Portuguese version of the Hospital Anxiety and Depression Scale. Psychol Health Med. 2007;12(2):225-35. http://dx.doi.org/10.1080/13548500500524088

25. Ferreira KA, Teixeira MJ, Mendonza TR, Cleeland CS. Validation of brief pain inventory to Brazilian patients with pain. Support Care Cancer. 2011;19(4):505-11. https://doi.org/10.1007/s00520010-0844-7 
26. Campolina AG, Bortoluzzo AB, Ferraz MB, Ciconelli RM. Validação da versão brasileira do questionário genérico de qualidade de vida short-form 6 dimensions (SF-6D Brasil). Ciênc. saúde coletiva. 2011;16(7):3103-10. http://dx.doi.org/10.1590/ s1413-81232011000800010

27. Taunay TCD, Gondim FAA, Macêdo DS, Moreira-Almeida A, Gurgel LA, Andrade LMS, et al. Validação da versão brasileira da escala de religiosidade de Duke (DUREL). Rev. psiquiatr. clín. 2012;39(4):130-5. http://dx.doi.org/10.1590/S010160832012000400003

28. Look JO, Schiffman EL, Truelove EL, Ahmad M. Reliability and validity of Axis I of the Research Diagnostic Criteria for Temporomandibular Disorders (RDC/TMD) with proposed revisions. J Oral Rehabil. 2010;37(10):744-59. http://dx.doi. org/10.1111/j.1365-2842.2010.02121.x

29. Campos JADB, Carrascosa AC, Loffredo LCM, Faria JB. Consistência interna e reprodutibilidade da versão em português do critério de diagnóstico na pesquisa para desordens temporomandibulares (RDC/TMD - Eixo II). Rev. bras. fisioter. 2007;11(6):451-9. http://dx.doi.org/10.1590/s141335552007000600006
30. Schiffman E, Ohrbach R, Truelove E, Look J, Anderson G, Goulet JP, et al. Diagnostic Criteria for Temporomandibular Disorders (DC/TMD) for Clinical and Research Applications: recommendations of the International RDC/TMD Consortium Network* and Orofacial Pain Special Interest Groupt. J Oral Facial Pain Headache. 2014;28(1):6-27. https://doi.org/10.11607/ jop.1151

31. Rolke R, Baron R, Maier C, Tölle TR, Treede D-R, Beyer A, et al. Corrigendum to "Quantitative sensory testing in the German Research Network on Neuropathic Pain (DFNS): Standardized protocol and reference values" [Pain 123 (2006) 231-243]. Pain. 2006;125(1):197. http://dx.doi.org/10.1016/j.pain.2006.09.025

32. Kennedy DL, Kemp HI, Ridout D, Yarnitsky D, Rice ASC. Reliability of conditioned pain modulation: a systematic review. Pain. 2016;157(11):2410-9. https://doi.org/10.1097/j. pain. 0000000000000689

33. Silva WS, Oliveira RF, Ribeiro SB, Silva IB, Araújo EM, Baptista AF. Screening for Structural Hemoglobin Variants in Bahia, Brazil Int. J. Environ. Res. Public Health. 2016;13(2):225. http://dx.doi. org/10.3390/ijerph13020225 